\title{
Evaluation of Antibacterial Effect of Silver Nonoparticle Coated Stainless Steel Band Material - An In vitro Study
}

\author{
Dr S Hima Bindu,, Dr S V Kala Vani,,2 Dr G Nirisha,3 Dr N Madhuri,4 \\ Dr B Sai Deepa, 5 Dr S Hemadrí \\ 'Clinical Practitioner, Smile Care Multispeciality Dental Clinic, ${ }^{2}$ Professor and HOD, \\ 3.6Post Graduate Student, Department of Orthodontics and Dentofacial Orthopaedics, \\ C.K.S. Theja Institute of Dental Sciences \& Research, ${ }^{4 C}$ linical Practitioner, ${ }^{5 C l}$ linical Practitioner, India
}

Correspondence: Dr. S.V. Kala Vani; Email: drsvkvaniortho@gmail.com

\section{ABSTRACT}

Introduction: Decalcification, caries, inflammatory periodontal disease are the most common iatrogenic effects of orthodontic treatment because of failure to maintain proper oral hygiene. Although various methods have been tried to minimize the incidence of white spot lesions, none of them proved to be effective. The purpose of this study was to develop a hard coating of silver nanoparticles on stainless steel band material and to evaluate the antibacterial efficacy against most common cariogenic pathogens.

Materials \& Method: Stainless steel band material was cut into 45 pieces of about $0.5 \times 1 \mathrm{~cm}$ in dimension, of these 25 band material strips were coated with silver nanoparticles using thermal evaporation technology in a Vacuum coating unit (Indovision, India) at a vacuum of $4.5 \times 10-5$ millibar at $961^{\circ} \mathrm{C}$ for 5 minutes and remaining strips served as control. Scanning electron microscopy (SEM) study of coated band material showed a uniform deposition of silver nanoparticles of about 18.63 percent by weight. Five coated and five uncoated band material strips were utilized for each test to evaluate the antibacterial effect of the coated band material against Streptococcus mutans, Lactobacillus acidophilus using zone of inhibition and direct contact test. In zone of inhibition test, the bacterial growth inhibition zone was measured after a period of 24-48 hours, where as in direct contact test, the number of bacterial colonies were counted after 24 hours, 48 hours and 1 week. Five coated band materials were immersed separately in a container having $5 \mathrm{ml}$ of artificial saliva and the amount of silver nanoparticles released from coated samples was evaluated after $24 \mathrm{hrs}, 48 \mathrm{hrs}$, and 1 week using atomic absorption spectrophotometer.

Result: A stable uniform coating of silver nanoparticles on the band material was obtained by physical vapor deposition. The coated band material showed a potent antibacterial activity against L.acidophilus and S.mutans. The maximum amount of silver nanoparticles released from the silver nanoparticle coated band material was $0.0236 \pm 0.0067 \mathrm{ppm}$, which is below the maximum permissible level set by WHO [0.1 mg /l], proving it as biocompatible.

Conclusion: Silver nanoparticle coating on orthodontic band surfaces can provide suitable antimicrobial activity during active orthodontic treatment.

Keywords: Antibacterial effect, Band material, Decalcification, Silver nanoparticles

\section{INTRODUCTION}

Fixed orthodontic treatment involves placement of bands and brackets on the teeth, and orthodontic bands are often seated in supra and subgingival areas. Banding of molar teeth has more tendencies for retention of plaque due to the greater area of band material compared to bonded attachments and also due to the obstruction caused by band material to oral prophylactic measures. Accumulation of plaque around bands results in decalcification, white spot lesions and gingival inflammation which may progress to periodontal disease. Poor oral hygiene is also shown to increase the orthodontic treatment time, as the cells involved in the tooth movement such as osteoclasts, do not efficiently perform in an inflammatory environment.'

Routine oral hygiene measures depend on patient compliance. Even higher concentration of chlorhexidine was not effective in reducing the Streptococcus mutans count. ${ }^{2}$ The issue of bacterial infection can be solved by adjusting the antimicrobial properties of a metal surface prior to appliance placement. Various techniques described in the 
literature include direct impregnation with antibiotics and the use of antibiotics or silver doped polymer coatings. ${ }^{3}$ Among various metals, silver, zinc, copper exhibits high antibacterial property. ${ }^{4}$ Nanoparticles (NP) have a greater surface-to-volume ratio (per unit mass) compared to non-nanoscale particles, interacting more closely with microbial membranes and provide considerably larger surface area for antimicrobial activity. ${ }^{5}$ The growing numbers of bacterial strains are becoming antibiotic-resistant and bacteria are less likely to develop resistance against metal NPs than conventional antibiotics. ${ }^{6-9}$

As silver nanoparticles (SNP) were known to have good antibacterial activity and biocompatibility, ${ }^{10}$ stainless steel band material was coated with silver nanoparticles. Various methods of coating were described in the literature, which includes thermal vacuum evaporation ${ }^{1,3,8}$ spray pyrolysis, ${ }^{13}$ high vacuum magnetron sputtering equipment14-16 silanization,? electroplating, ${ }^{11}$ dip coating method. ${ }^{17}$ In the present study, stainless steel bands are coated with silver nanoparticles using thermal vacuum evaporation technology because this method is capable of producing very high purity thin film with high deposition rate and damage to the substrate can be minimized. Antibacterial efficacy of silver nanoparticle coated band material against most common cariogenic pathogens was evaluated.

\section{MATERIALS AND METHOD}

Stainless steel band material (0.005"' $\left.\times 0.018^{\prime \prime}\right)$ was cut into 45 pieces of about $0.5 \times 1 \mathrm{~cm}$ in dimension. Twenty five stainless steel strips were coated with silver nanoparticles and included in the test group; the remaining strips served as controls. [Table1]

The coating was done by thermal evaporation technique using - Vacuum coating unit (Indovision, India) at a vacuum of $4.5 \times 10-5$ millibar at $961^{\circ} \mathrm{C}$ for 5 minutes. The substrate (band material) temperature was maintained at $100^{\circ} \mathrm{C}$. The silver wire was kept over tungsten filament at a distance of $15 \mathrm{~cm}$ from the band material and vaporized to form a uniform coating of silver nanoparticles on the band material.

\section{Characterization of the coated surface}

The surface morphology of the coated and uncoated samples was examined by scanning electron microscopy (SEM) and the atomic composition was determined by energy-dispersive $\mathrm{x}$-ray spectroscopy (EDS).

\section{Antimicrobial activity test}

The antibacterial properties of the samples were evaluated against Gram-positive S.mutans and L.acidophilus using zone of inhibition test and direct contact test.

\section{Zone of inhibition test}

The medium (brain heart infusion agar for Lactobacillus and mutans sanguis agar for S.mutans) (HiMedia, Mumbai) was prepared from dehydrated media as per manufacturer's instructions. Medium at pH 7.2 to 7.4 was transferred to $9 \mathrm{~cm}$ diameter Petri dishes and stored at $2-8^{\circ} \mathrm{C}$. Lactobacillus and S.mutans suspensions were prepared in $0.5 \mathrm{McF}$ arland standard concentration (108 bacteria per $\mathrm{mL}$ ) and transferred to culture medium. Each coated (test sample) and uncoated (control sample) band were placed in the culture using sterile forceps at specific millimeter distance, and all five plates were incubated for 2448 hours at $37^{\circ} \mathrm{C}$. Then the bacterial growth inhibition zone was measured in millimeters.

\section{Direct contact test}

Five coated and five uncoated band materials were placed in separate micro-tubes containing $1 \mathrm{~mL}$ of brain heart infusion broth for Lactobacillus and mutans sanguis broth for S.mutans. When the strains reached

Table 1.Distribution of samples

\begin{tabular}{|l|l|c|c|}
\hline \multicolumn{2}{|c|}{ Total number of sample ( $\mathbf{n}) \mathbf{4 5}$} \\
\hline \multirow{2}{*}{ Lactobacillus } & \multicolumn{1}{|c|}{ Disk diffusion test } & Control sample (nc) - 20 & Test sample ( $\mathbf{n t )} \mathbf{- 2 5}$ \\
\cline { 2 - 4 } & Direct contact test & 5 & 5 \\
\hline \multirow{2}{*}{ Streptococcus } & Disk diffusion test & 5 & 5 \\
\cline { 2 - 4 } & Direct contact test & 5 & 5 \\
\hline Amount of silver ion released & - & 5 & 5 \\
\hline
\end{tabular}


the standard 0.5 McFarland concentration, they were diluted in 1:10 ratio and $5 \mu \mathrm{L}$ of this suspension were then poured into each tube containing $1 \mathrm{~mL}$ of the culture medium. The microtubes were incubated at $37^{\circ} \mathrm{C}$ and after a period of 24 hours, 48 hours and 1 week, ten $\mu \mathrm{L}$ of the suspension were taken from each microtube (five coated, and five uncoated) using a micropipette and cultured on a separate culture medium. These cultures were incubated at $37^{\circ} \mathrm{C}$ for $24-$ 48 hours and the colonies, each composed of a single set of cultures, were counted.

\section{Amount of silver ion released}

Five coated band materials were immersed separately in a container having $5 \mathrm{ml}$ of artificial saliva with gentle shaking. The saliva was replaced and analyzed after 24 hours, 48 hours, and 1 week. The amount of silver ions leached from the coated samples were measured using atomic absorption spectrophotometer.

Data obtained from the results were analyzed statistically using the paired t-test for intragroup comparison of test and control and independent t-test for intergroup comparison.

\section{RESULT}

\section{Surface morphology and atomic composition}

SEM images showed a uniform deposition of silver nanoparticleson coated band material, whereas uncoated sample showed only generic metal striations (Fig.1a, 1b). The EDS spectra of the uncoated sample showed elemental ion content of typical bands such as $\mathrm{Cr}$, Fe, and Ni. However, no silver ions were detected. EDS spectra of the coated stainless steel band material showed the presence of silver ions in addition to the typical composition of the steel. The amount of Ag nanoparticles deposited on the coated band material was about 18.63 percent by weight. (Fig. 2a, 2b)

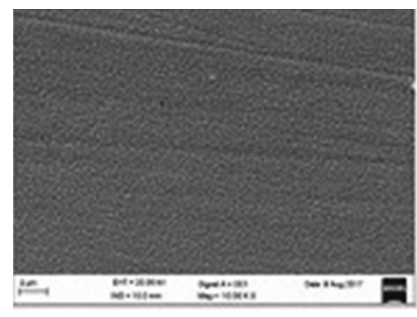

Fig. 1a

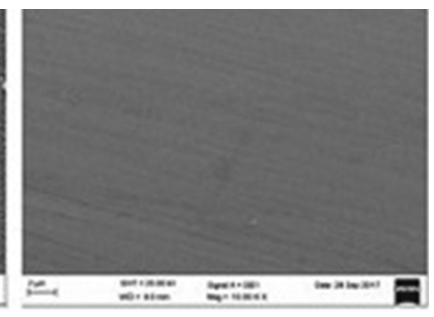

Fig. 1b
Figure 1: SEM images coated (a) and uncoated (b) band material

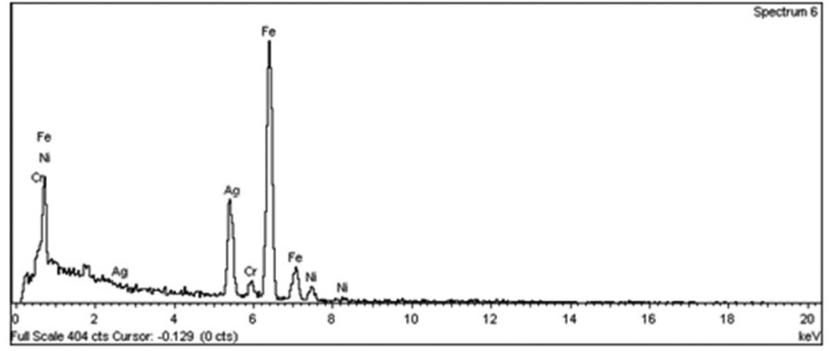

Figure 2a: Image showing elemental composition of coated

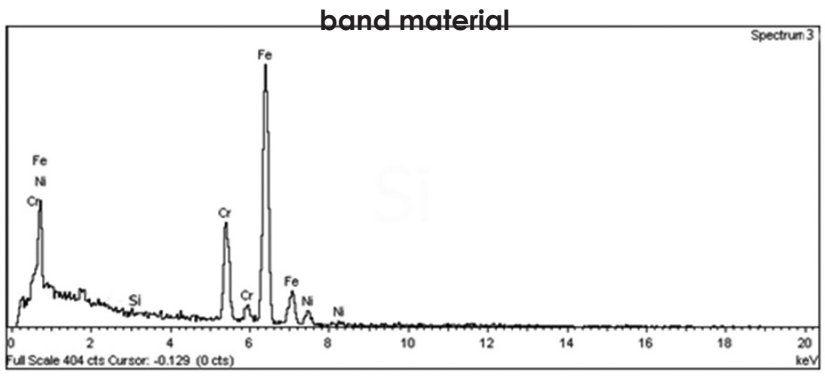

Figure $2 \mathrm{~b}$ : Image showing elemental composition of uncoated band material

\section{Antibacterial effect}

\section{Zone of inhibition test}

A clear zone of inhibition of about $4 \mathrm{~mm}$ was observed around the coated samples against both L.acidophilus and S. mutans. Whereas, inhibition zone was not observed around the uncoated SS strips. (Fig. 3a,3b) [Table 2]

Table 2: Comparison of the zone of inhibition values between control and test groups against Lactobacillus and Streptococcus using disk diffusion assay.

\begin{tabular}{|l|c|c|c|c|c|}
\hline \multirow{2}{*}{ Group } & \multirow{2}{*}{ N } & \multicolumn{2}{c|}{ Lactobacillus } & \multicolumn{2}{c|}{ Streptococcus } \\
\cline { 3 - 6 } & & Mean & SD & Mean & SD \\
\hline Control & 5 & $0.00 \mathrm{~mm}$ & 0.000 & $0.00 \mathrm{~mm}$ & 0.000 \\
\hline Test & 5 & $4.300 \mathrm{~mm}$ & .2739 & $4.000 \mathrm{~mm}$ & .3536 \\
\hline Mean Difference & \multicolumn{2}{|c|}{-4.3} & \multicolumn{2}{|c|}{-4} \\
\hline p value & \multicolumn{2}{|c|}{$<0.001^{* *}$} & \multicolumn{2}{c|}{$<0.001^{* *}$} \\
\hline
\end{tabular}

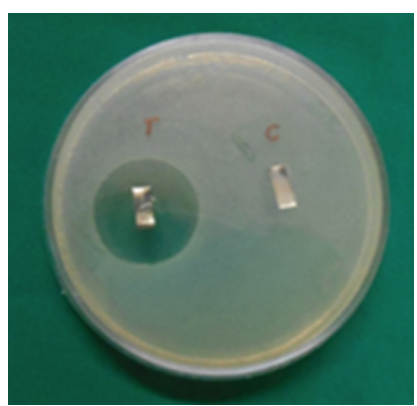

Fig. 3a

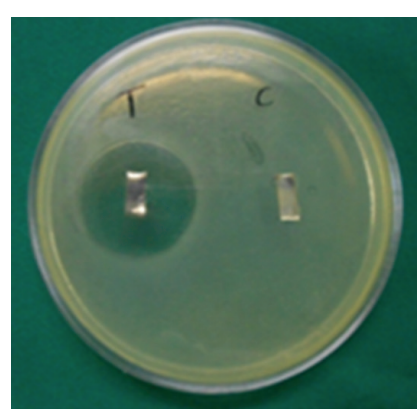

Fig. $3 b$
Figure 3: Petridishes showing zone of inhibition against (a) Lactobacillus (b) Streptoccous 
Table 3: Intergroup comparison of the zone of inhibition against Lactobacillus and Streptococcus in test and control groups using disk diffusion assay.

\begin{tabular}{|l|c|c|c|c|c|c|}
\hline \multirow{2}{*}{ Parameter } & \multirow{2}{*}{ N } & \multicolumn{2}{|c|}{ Lactobacillus } & \multicolumn{2}{c|}{ Streptococcus } & \multicolumn{2}{c|}{ Mean } \\
\cline { 2 - 6 } & & Mean & SD & Mean & SD & Difference \\
\hline Control & 5 & $0.00 \mathrm{~mm}$ & 0.000 & $0.00 \mathrm{~mm}$ & 0.000 & - \\
\hline Test & 5 & $4.300 \mathrm{~mm}$ & 0.2739 & $4.000 \mathrm{~mm}$ & 0.3536 & $0.172 \mathrm{NS}$ \\
\hline
\end{tabular}

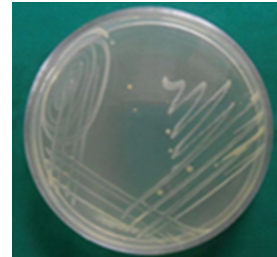

Fig. $4 a$

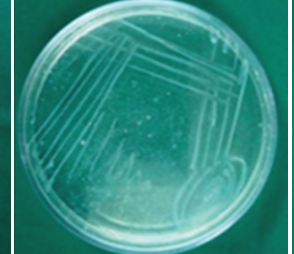

Fig. $4 b$

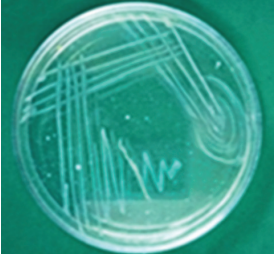

Fig. 4c

Figure 4: Petridishes showing Lactobacillus colonies of control samples at (a) 24 hours, (b) 48 hours and (c) 1 week

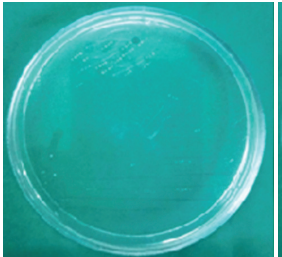

Fig. $5 a$

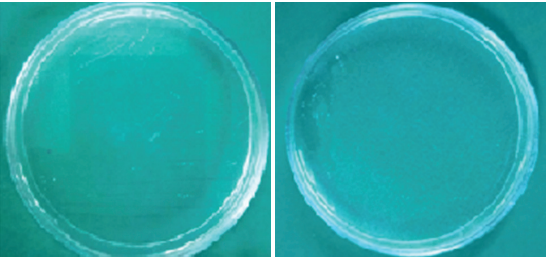

Fig. 5b
Fig. 5c
Figure 5: Petridishes showing Lactobacillus colonies of test samples at (a) 24 hours, (b) 48 hours and (c) 1 week

Table 4: Comparison of the number of Lactobacillus coloniesin test and control groups at different time intervals using a direct contact test

\begin{tabular}{|c|c|c|c|c|c|c|c|}
\hline \multirow{2}{*}{ Duration } & \multirow{2}{*}{$\mathbf{N}$} & \multicolumn{2}{|c|}{ Control } & \multicolumn{2}{|c|}{ Test } & \multirow{2}{*}{ Mean difference } & \multirow{2}{*}{$P$ value } \\
\hline & & Mean & SD & Mean & SD & & \\
\hline 24 hours & 5 & 220.80 & 4.147 & 44.60 & 3.209 & 176.2 & $<0.001^{* *}$ \\
\hline 48 hours & 5 & 323.60 & 12.033 & 24.40 & 2.966 & 299.2 & $<0.001^{* *}$ \\
\hline 1 week & 5 & 451.00 & 5.657 & 19.00 & 2.646 & 432.2 & $<0.001^{* *}$ \\
\hline
\end{tabular}

\section{Direct contact test}

The mean number of Lactobacillus colonies grown after 24 and 48 hours and 1 week continued to increase in the control group but has decreased in the test group which was statistically significant. (Fig. 4, 5) [Table 4]

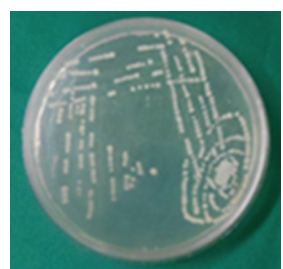

Fig. 6a

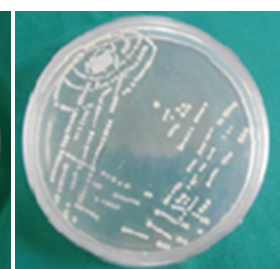

Fig. 6b

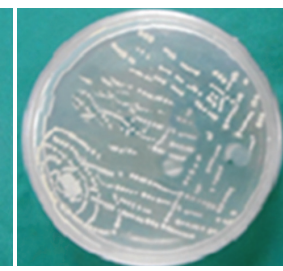

Fig. 6c
Figure 6: Petridishes showing streptococcus mutans colonies of control group at (a) 24 hours, (b) 48 hours and (c) 1 week
The mean number of Streptococcus colonies also showed a similar pattern and the difference between control and test groups after 24 hours, 48 hours and 1 week was statistically significant. (Fig. 6, 7) [Table 5]

Table 5: Comparison of the number of Streptococcus colonies in test and control groups at various duration using a direct contact test

\begin{tabular}{|l|c|c|c|c|c|c|c|}
\hline \multirow{2}{*}{ Duration } & \multirow{2}{*}{ N } & \multicolumn{2}{|c|}{ Control } & \multicolumn{2}{c|}{ Test } & Mean difference & \multirow{2}{*}{ P value } \\
\cline { 2 - 8 } & & Mean & SD & Mean & SD & 209.6 & $<0.001^{* *}$ \\
\hline 24 hours & 5 & 254.40 & 6.542 & 44.80 & 2.775 & 301.4 & $<0.001^{* *}$ \\
\hline 48 hours & 5 & 333.60 & 9.711 & 32.20 & 3.033 & 504.2 & $<0.001^{* *}$ \\
\hline 1 week & 5 & 522.80 & 14.755 & 18.60 & 3.050 & \\
\hline
\end{tabular}

Table 6: Comparison of silver nanoparticles release in the test group at different time intervals.

\begin{tabular}{|l|c|c|c|c|}
\hline \multicolumn{1}{|c|}{ Duration } & N & Mean & SD & P value \\
\hline at 24 hours & 5 & $.0236800 \mathrm{ppm}$ & .0067937 & \\
\cline { 1 - 4 } at 48 hours & 5 & $.0221720 \mathrm{ppm}$ & .0056420 & $<0.001^{* *}$ \\
\hline at 1 week & 5 & $.0160060 \mathrm{ppm}$ & .0032339 & \\
\hline
\end{tabular}




\section{Measurement of silver ion released}

There was a significant decrease in the concentration of silver ions released from coated samples at 24 hours, 48 hours and 1 week with maximum release of 0.0236 $\pm 0.0067 \mathrm{ppm}$ at 24 hours, which is less than maximum permissible levels set by WHO $(0.1 \mathrm{mg} / \mathrm{L})$. The mean concentration of silver ions released at 24 hours, 48 hours, and 1 week were $0.0236 \pm 0.0067 \mathrm{ppm}, 0.0221 \pm$ $0.0056 \mathrm{ppm}, 0.016 \pm 0.0032 \mathrm{ppm}$ respectively. [Table 6]

The maximum silver ion release of about $0.0236 \pm 0.0067$ ppm was noted at $24 \mathrm{hrs}$ which is less than maximum permissible level set by WHO (0.1 ppm ). ${ }^{19}$

\section{DISCUSSION}

White spot formation or demineralization is a prevalent unwanted side-effect of orthodontic therapy. Orthodontic appliances can affect the self-cleaning ability of teeth, alter the oral microflora and increase the levels of acidogenic plaque bacteria, i.e. mutans streptococci and lactobacilli in saliva and dental biofilm during active wear of the appliance.

In the oral cavity, the antibacterial properties of NPs have been used through two broad mechanisms of combining dental materials with NPs or coating surfaces with NPs to prevent microbial adhesion, with the overall aim of reducing the biofilm formation. ${ }^{6}$ An antibacterial coating on orthodontic appliances offers a possible strategy for reducing such bacterial damage.

The present study was designed with reference to the unique antimicrobial properties of silver nanoparticles. In this study, stainless steel band material used to band molar teeth were coated with silver nanoparticles to produce a potentially antimicrobial band material effective against Gram-positive bacteria.

The coating technique used in this study was vacuum evaporation technology. Vacuum evaporation is a physical deposition method that uses resistive heating to produce a metallic thin film of solid on a suitable substrate. The SEM images of coated and uncoated band materials were compared to assess the presence of surface SNPS. SEM images of coated band material showed uniform deposition of SNPs, whereas uncoated band material showed only generic metal striations. EDS for coated surface showed the presence of silver nanoparticles.
The amount of silver nanoparticles deposited as reported in various studies ranged from $4.26 \%-100$ $\%$ by weight. Juan et al ${ }^{9}$ deposited $4.26 \%$ of silver on the titanium surface using silanization method, they found negligible zones of inhibition against E.coli and S.aureus, whereas study by Prabha et al deposited $28.22 \%$ of silver on the band material surface using thermal vacuum evaporation technology and evaluated cytotoxicity with 3-(4, 5-dimethyl thiazol-2yl)-2, 5-diphenyl tetrazolium bromide (MTT) assay which shows that more than 80 percent viability in all coated samples.' In our present study, approximately $18.63 \%$ of silver nanoparticles by weight was deposited on the stainless steel band material, which is thought to have better antibacterial effect and biocompatibility.

The results of our study showed a clear zone of inhibition of $4.3 \mathrm{~mm}$ and $4.0 \mathrm{~mm}$ diameter around coated band material against L.acidophilus and S.mutans respectively, whereas there is no growth inhibition zone around uncoated band material. In contrast, Prabha et al' noted no growth inhibition around the silver coated band material against S.mutans. Arash et al" evaluated the antibacterial effect of silver (8 - $10 \mu \mathrm{m}$ ) coated bracket against S.mutans using disk diffusion assay. They showed that the inhibition halo was absent around the silver coated bracket, which was attributed to smaller physical contact between the silver particles in the bracket and the surrounding culture medium. Morita et $\mathrm{al}^{10}$ noted a clear zone of inhibition of more than $4.2 \mathrm{~mm}$ diameter around $\mathrm{Ag}$ ion-coated retainer against $S$.mutans indicating that Ag ions on the coated wires diffused into the culture medium and inhibited bacterial growth. No studies in the literature reported the antibacterial effect of silver nanoparticles against Lactobacillus using disk diffusion assay. There was no difference in the antibacterial effect of silver nanoparticles against L.acidophilus and S.mutans using disk diffusion assay. [Table.3]

In the direct contact test, the number of bacterial colonies continued to decrease in the test group, which was attributed to the potent antibacterial effect of silver nanoparticles against both S.mutans and L.acidophilus. The results were similar to the study by Arash et al $^{11}$ in which S.mutans colonies increased slightly after six hours and subsequently decreased against silver $(8-10 \mu \mathrm{m})$ coated SS brackets when evaluated for a period of 30 days. A study by Mhaske et $\mathrm{al}^{18}$ showed a significant decrease in the total 
number of lactobacillus colonies around silver coated SS wire (10 $\mathrm{nm}$ thick) compared with the control group after 24 hours when evaluated using direct contact test. The results of our study showed that there is no significant difference in the number of L.acidophilus and S.mutans colonies in the test group at 24 hours and 1 week. This implies that silver nanoparticles exhibited similar antibacterial effect against both L.acidophilus and S.mutans.

In this study, the amount of silver ions released from coated band material continued to decrease over a period of 1 week, with a maximum release of $0.023 \pm$ $0.0067 \mathrm{ppm}$ at 24 hours. This was less than the maximum permissible levels set by the WHO $(0.1 \mathrm{mg} / \mathrm{L}) .{ }^{19}$ No studies in the literature evaluated the concentration of silver ions released from the coated band material over a period of 1 week. However, there are other studies in literature, in which the silver ions release were evaluated from silver nanoparticles coated retainers and stainless steel sheets.

Rahmani et $\mathrm{al}^{8}$ noted that the amount of silver ions released from silver coated $(200 \mu \mathrm{m})$ retainers using PVD after 24 hours of immersion in artificial saliva as $0.029 \pm$ 0.005 ppm. Morita et $\mathrm{al}^{10}$ reported the amount of silver ions released from silver coated $(14.817 \pm 2.163 \mu \mathrm{m})$ titanium retainer after 24 hours as $0.043 \pm 0.005$ ppm. Chen et $\mathrm{al}^{12}$ developed a silver coated $(2.68 \mathrm{\mu g} /$ $\mathrm{cm} 2$ ) stainless steel strip using a chemical method and evaluated the amount silver ion released after $6,12,24,36$ and 48 hours. They observed that the amount of silver ion released at 24 hours was 0.07 ppm and silver ion release rate during the first 24 hours is lower than in the next 24 hours. SogRyuet al3) measured the amount of silver ion released from silver coated ( $100 \%$ by weight) stainless steel strip after 1st, 2nd, and 8th day. The concentration of $\mathrm{Ag}$ ions released after 2nd and 8th day was 1.2ppm, 1.95ppm respectively.
They reported that the silver ion concentration on the 8thday was more than the 2 nd day, as the solution was not replaced after the 2 nd day.

Although nanoparticle coating was done on different materials for different purposes, in studies reported so far, only one study reported silver nanoparticle coating on stainless steel band material. Our data cannot be compared with other studies because of difference in the amount of silver nanoparticle deposited on the surface, the method of coating used, size and surface characteristics of a sample on which coating was done.

As the orthodontic treatment lasts for about 12-24 months, it is pertinent to carry out long term studies to establish standard protocols which would facilitate continuous release of nanoparticles from coated band material in order to maintain prolonged antibacterial activity.

Since the in vitro studies can never simulate oral conditions precisely. Further clinical trials are required to study the antibacterial effect, biocompatibility.

\section{CONCLUSION}

In this short term study, band material coated with silver nanoparticles showed a good antibacterial effect against L.acidophilus and S.mutans for a period of one week. This silver nanoparticle coated band material is especially useful in cases where orthodontic treatment lasts for longer duration such as cleft cases and orthognathic surgery and in individuals who lacks manual dexterity.

\section{Financial support and sponsorship: Nil.}

Conflicts of interest: There are no conflicts of interest. 


\section{REFERENCES}

1. Prabha RD, Kandaswamy R, Sivaraman US, Nandakumar MA. Antibacterial nanosilver coated orthodontic bands with potentia implications in dentistry. Indian J Med Res. 2016; 144(4): 580-586.

2. Attin R, Ilse A, Werner C, Wiegand A, Attin T. Antimicrobial effectiveness of a highly concentrated chlorhexidine varnish treatment in teenagers with fixed orthodontic appliances. Angle Orthod2006; 76: 1022-7.

3. Ryu HS, Bae IH, Lee KG, Hwang HS. Antibacterial effect of silver - platinum coating for orthodontic appliances. Angle Orthod.2012; 82 (1): 151-7.

4. Yasuyuki M, Kunihiro K, Kurissery S. Antibacterial properties of nine pure metals: laboratory study using Staphylococcus aureus and Escherichia coli. Biofouling.2010; 26(7):851-8.

5. Pal S, Tak YK, Song JM. Does the antibacterial activity of silver nanoparticles depend on the shape of the nanoparticle? A study of the Gram-negative bacterium Escherichia coli. Appl Environ Microbiol 2007;73:1712-20.

6. Correa JM, Mori M, Sanches HL, Cruz AD, PoiateE.Silver Nanoparticles in Dental Biomaterials. International Journal of Biomaterials 2015.

7. Farhani AB, Borzabadi E, L E. Nanoparticles in orthodontics, a review of antimicrobial and anti-caries applications. ActaOdontologica Scandinavica. 2013

8. Rahmani A, Arash V, Ghorbanipour R, Rabiee SM, Bijani A. Clinical, Histological and Histomorphometric evaluation of effects of silver ion coating of orthodontic fixed retainers on gingival health in rabbits. Biomed Pharmacol J 2017; 10(2).

9. Juan L, Zhimin Z, Anchun M. Deposition of silver nanoparticles on titanium surface for antibacterial effect. Int J Nanomedicine. 2010; 5 : $261-267$.

10. Morita Y, Imai S, Hanyuda A, Matin K, Hanada N, Nakamura Y. Effect of silver ion coating of fixed orthodontic retainers on the growth of oral pathogenic bacteria. Dent Mater J 2014; 33(2): 268-274.

11. Arash V, Keikhaee F, Rabiee SM, Rajabnia R, Khafri S. Evaluation of antibacterial effects of silver-coated stainless steel orthodontic brackets. J Dent (Tehran). 2016; 13 (1):49-54.

12. Chen L, Boughton R. Chemical assembly of silver nanoparticles on stainless steel for antimicrobial applications. Surface \& Coatings Technology 2010; 204 (23): 3871- 75.

13. Ramazanzadeh B, Jahanbin A, Yaghoubi M, Ghazvini K, Shafaee H .Comparison of antibacterial effects of ZnO and CuO nanoparticles coated brackets against streptococcus mutans.J Dent (Shiraz).2015; 16(3):200-205.

14. Huang HL, Chang YY, Lai MC, Lin CR, Shieh TM. Antibacterial TaN-Ag coatings on titanium dental implants. Surface \& Coatings Technology 2010; 205 (5): $1636-41$.

15. Chang YY, Lai CH, Hsu JT, Tang CH, Liao WC, Huang HL. Antibacterial properties and human gingival fibroblast cellcompatibility of TiO2/ Ag compound coatings and ZnO films on the titanium-basedmaterial.Clin Oral Invest 2012; 16(1):95-100.

16. Cao B, Wang Y, Li N, Liu B, Zhang Y.Preparation of an orthodontic bracket coated with a nitrogen-doped TiO2-xNy thin film and examination of its antimicrobial performance. Dent Mater J 2013; 32(2): 311-6.

17. Syed SS, Kulkarni D, Todkar R, Bagul RS, Parekh K, Bhujbal N. A novel method of coating orthodontic arch wires with nanoparticles. J Int Oral Health.2015; 7 (5): 30-33.

18. MhaskecAR, Shetty PC, Bhat NS, Chandra CSR, Laxminath SM. Antiadherent and antibacterial properties of stainless steel and NiTi orthodontic wires coated with silver against Lactobacillus acidophilus—an in vitro study. Prog.Orthod.2015; 16:40.

19. Pelkonen KH, Heinonen-Tanski H, Hänninen. Accumulation of silver from drinking water into cerebellum and musculus soleus in mice. Toxicology 2003; 186: 151-157. 